\title{
Fluoxetine synergizes with temozolomide to induce the CHOP-dependent endoplasmic reticulum stress-related apoptosis pathway in glioma cells
}

\author{
JIAN MA $^{1 *}$, YAN-RU YANG $^{1 *}$, WEI CHEN $^{1}$, MEI-HUA CHEN $^{1}$, HAO WANG $^{1}$, \\ XIAO-DAN WANG ${ }^{1}$, LI-LI SUN ${ }^{1}$, FENG-ZE WANG $^{2}$ and DE-CAI WANG ${ }^{1}$ \\ ${ }^{1}$ School of Pharmaceutical Science and ${ }^{2}$ School of Life Science, Taishan Medical University, \\ Taian, Shandong 271016, P.R. China
}

Received January 9, 2016; Accepted February 18, 2016

DOI: $10.3892 /$ or.2016.4860

\begin{abstract}
Although temozolomide (TMZ) is the most effective chemotherapy agent for glioma, chemotherapy resistance has limited its clinical use. Fluoxetine (FLT), which is widely used in cancer-related depression, has exhibited potent anticancer properties in different cancer cell types. The aim of this study was i) to evaluate the antitumor mechanism of FLT, and ii) to further evaluate the effects of a combination of FLT and TMZ on glioma cells. Glioma cell lines were exposed to FLT and/or TMZ. Cell viability and apoptosis were examined by CCK- 8 assay, flow cytometry and caspase- 3 activity assay, respectively. The expression of endoplasmic reticulum-stress (ERS) apoptosis-related proteins was measured using realtime PCR and western blotting. Synergism between the two drugs was evaluated by the combination index (CI) through CompuSyn software. FLT significantly and dose-dependently inhibited the proliferation of various glioma cell lines, and rat glioma C6 cells had a highly sensitive response to the addition of FLT. FLT treatment increased the early apoptosis rate, induced typical apoptotic morphology in the C6 cells and activated caspase- 3 with no change in the mitochondrial membrane potential. Further study showed that FLT activated the ERS marker, CHOP. This induction was associated with activation of the PERK-eIF2 $\alpha$-ATF4 and ATF6 cascade. Concomitantly, GADD34, a downstream molecule of CHOP, was also increased. Combined FLT and TMZ treatment showed a synergistic cytotoxic effect in the C6 glioma cells. Knockdown of CHOP expression abolished the synergistic effect of FLT and TMZ in the C6 cells, which suggests that FLT
\end{abstract}

Correspondence to: Professor De-Cai Wang, School of Pharmaceutical Science, Taishan Medical University, 619 Changcheng Road, Taian, Shandong 271016, P.R. China

E-mail: dcwang@tsmc.edu.cn

${ }^{*}$ Contributed equally

Key words: glioma, fluoxetine, temozolomide, apoptosis, CHOP may sensitize glioma cells to TMZ through activation of the CHOP-dependent apoptosis pathway. These results revealed that FLT induced glioma cell apoptosis and sensitized glioma cells to TMZ through activation of the CHOP-dependent apoptosis pathway. The present study provides a primary basis for using the combination of these drugs in patients with advanced glioma.

\section{Introduction}

Gliomas are the most common primary brain tumors, and they constitute approximately $30 \%$ of all brain and central nervous system tumors and $80 \%$ of all malignant brain tumors (1). Less than $3 \%$ of glioma patients are still alive at 5 years after diagnosis (2). Treatment for brain gliomas is a combined approach, using surgery, radiation therapy and chemotherapy. However, limited by the location of the tumor, glioma is difficult to be resected completely by surgery. As a result, radiotherapy and chemotherapy are necessary to increase the survival time of patients $(3,4)$.

Temozolomide (TMZ), which is able to cross the bloodbrain barrier effectively, has become the most effective chemotherapy agent for glioma. A clinical study demonstrated that radiotherapy and adjuvant TMZ treatment leads to an increased median survival time of approximately 14.6 months $(5,6)$. Unfortunately, however, the response rate of TMZ treatment is not satisfactory, and the response is usually accompanied by chemotherapy resistance and high rates of toxicity such as severe nausea, vomiting, genotoxic, teratogenic and fetotoxic effects (7). There has been a shift from management with traditional, non-specific cytotoxic chemotherapies to treatment with molecular-specific targeted therapies that are used either alone or in combination with traditional chemotherapy and radiation therapy. Thus, more effective and less toxic agents for glioma are urgently required. Researchers have conducted laboratory and clinical studies to investigate whether it may be possible to enhance the anticancer potency of TMZ by combination with other pharmacologic agents. In preclinical trials, it was found that chloroquine increased the chemosensitivity of glioma cells to TMZ (8). A laboratory study indicated that TMZ killed brain tumor cells more effi- 
ciently when epigallocatechin gallate (EGCG), a component of green tea, was added (9). More recently, use of the novel oxygen diffusion-enhancing compound trans sodium crocetinate (TSC) when combined with TMZ and radiation therapy has been investigated in preclinical studies and a clinical trial is currently underway (10).

Clinical studies have shown that depressive disorder is common among patients with advanced cancer, and anti-depression treatment in cancer patients not only improves depressive symptoms, but also enhances immunologic function, thereby improving the quality of life of cancer patients $(11,12)$. Thus, anti-depressants are routinely prescribed to cancer patients with depression. As a representative of selective serotonin reuptake inhibitors (SSRIs), fluoxetine (FLT) is one of the most commonly used anti-depressants (13). While increased attention has been given to the direct effects of antidepressants on tumor cells, recent preclinical studies suggest that the use of FLT may be associated with a reduced colon cancer risk (14-16). Actually, a growing body of evidence has demonstrated that FLT possesses antitumor activity in different cancer cell types, such as hepatocellular carcinoma (17), OC2 human oral cancer (18) and human epithelial ovarian cancer OVCAR-3 cell lines (19). In addition, FLT was also found to have a synergistic effect with anticancer drugs and can overcome drug resistance (20-22). However, the downstream apoptotic signaling pathway involved in FLT-induced cell death remains unknown.

In the present study, we determined the in vitro cytotoxicity of FLT and explored the underlying mechanisms involved in its effects against glioma cells, and assessed the potential synergism of FLT and TMZ in inhibiting the growth of C6 glioma cells. These findings may provide a new therapeutic strategy to achieve anti-glioma synergism.

\section{Materials and methods}

Chemicals and antibodies. FLT and TMZ were obtained from Sigma-Aldrich (St. Louis, MO, USA). The antibodies against phospho-PERK, CHOP and caspase-3 were obtained from Cell Signaling Technology (Danvers, MA, USA), The antibodies against PERK, eIF2 $\alpha$, phospho-eIF2 $\alpha$, ATF4, ATF6 and GADD34 were obtained from Abcam (Cambridge, UK). The antibody against $\beta$-actin and horseradish peroxidaseconjugated anti-mouse or anti-rabbit IgG were obtained from Santa Cruz Biotechnology (Santa Cruz, CA, USA).

Cell culture. Cells were purchased from the Chinese Academy of Sciences Cell Bank (Shanghai, China). The cells were routinely cultured in Dulbecco's modified Eagle's medium (DMEM) supplemented with $10 \%$ fetal bovine serum and $100 \mathrm{U} / \mathrm{ml}$ penicillin and streptomycin (all from Invitrogen, Carlsbad, CA, USA) in a humidified incubator with $5 \% \mathrm{CO}_{2}$ at $37^{\circ} \mathrm{C}$.

CCK-8 assay. Cell viability assay was analyzed using a Cell Counting Kit-8 (CCK-8) (Dojindo Laboratories, Kumamoto, Japan). Briefly, the cells were plated on a 96-well culture plate at a density of 6,000 cells/well and were cultured overnight. The cells were then incubated in fresh culture medium containing FLT and/or TMZ at various concentrations for $24 \mathrm{~h}$. The cell viability was then assessed according to the manufacturer's instructions.

Apoptosis assay. C6 cells were seeded in 96-well microplates at 3,500 cells/well and treated with FLT for $24 \mathrm{~h}$. Then, $50 \mu \mathrm{l}$ of MitoTracker/Hoechst solution (Cellomics, Pittsburgh, PA, USA) was added to each well, and the cells were incubated at $37^{\circ} \mathrm{C}$ for $30 \mathrm{~min}$. Next, the cells were fixed with $100 \mu \mathrm{l}$ of the fixation solution, without removing the medium and the incubation continued in a fume hood at room temperature for $10 \mathrm{~min}$. The plate was sealed after being washed once and was run on the ArrayScan high-content screening (HCS) reader (Cellomics). The nuclear size and intensity of Hoechst fluorescence were also calculated and analyzed by Cell Motility BioApplication software.

Flow cytometric analysis. C6 cells were seeded at $4.0 \times 10^{5}$ cells/ well in 6-well plates and treated with FLT for $24 \mathrm{~h}$, and then washed twice with cold PBS and re-suspended at a concentration of $1 \times 10^{6}$ cells/ml. Next, $5 \mu$ l FITC-Annexin V and $10 \mu \mathrm{l}$ PI (both from BD Biosciences) were added into $100 \mu \mathrm{l}$ of the solution. After that, the cells were mixed gently and incubated for $15 \mathrm{~min}$ at room temperature in the dark. Finally, $1 \mathrm{ml}$ PBS was added and the samples were detected by flow cytometric analysis (Beckman Coulter, Brea, CA, USA) within $1 \mathrm{~h}$.

Caspase-3 activity measurements. C6 cells were seeded on a 96-well microplate at a density of 3,000 cells/well and treated with FLT at $24 \mathrm{~h}$. Then, $100 \mu \mathrm{l}$ of Caspase-Glo ${ }^{\circledR}$ 3/7 reagent (Promega, Madison, WI, USA) was added to each sample after allowing the plates to equilibrate at room temperature. The contents of the wells were gently mixed for $0.5-2 \mathrm{~min}$ and incubated at room temperature for $1 \mathrm{~h}$. The luminescence intensities were then analyzed using a multimode reader (Tecan, Männedorf, Switzerland) and the enzyme activities were expressed as relative luminescence units.

Measurement of the mitochondrial membrane potential. C6 cells were seeded on a 96-well plate at a density of 3,000 cells/ well and exposed to the drugs for $24 \mathrm{~h}$. Fifty microliters of MitoTracker/Hoechst solution (Cellomics Inc.) was added to each well, and the cells were incubated for $30 \mathrm{~min}$ at $37^{\circ} \mathrm{C}$. Next, we added $100 \mu \mathrm{l}$ of the fixation solution directly to each well without removing the medium and incubation was carried out in a fume hood at room temperature for $10 \mathrm{~min}$. Then we aspirated the supernatant and washed the plate once with $150 \mu 1$ wash buffer. Images were acquired on the ArrayScan ${ }^{\circledR}$ HCS system; the intensity of red fluorescence represented the MMP of the cells.

Quantitative real-time $R T-P C R$. C6 cells were plated on a 6 -well plate at a density of $4 \times 10^{5}$ cells/well, and exposed to the drugs for $24 \mathrm{~h}$. Total RNA extraction was isolated from the cells using an RNeasy Mini kit (Qiagen, Valencia, CA, USA). The first strand cDNA synthesis was carried out using RevertAid $^{\mathrm{TM}}$ First Strand cDNA Synthesis kit (Fermentas, Burlington, CA, USA). The RT mixture was mixed with Quanti Fast SYBR Green PCR Kit (Qiagen, Hilden, Germany) and gene-specific primers in a final volume of $20 \mu \mathrm{l}$. The following oligonucleotide primers were used as specific for the 
gene CHOP (5'-CTGGAAGCCTGGTATGAGGA-3' and 5'-AGG TGCTTGTGACCTCTGCT-3') and gene GADD34 (5'-CCT TGATGTGGAAGCCCAAAGTT-3' and 5'-TCCACTTCT TGCTCTCTAAGGCCAT-3') (Invitrogen). The expression levels of the genes were normalized with that of $\beta$-actin (5'-CCCATCTATGAGGGTTACGC-3' and 5'-TTAATGTCA CGCACGATTTC-3') housekeeping gene product as an endogenous reference. The quantitative real-time RT-PCR was performed in ABI 7500 Fast Real-Time PCR (Life Technologies, Carlsbad, CA, USA). The amplification conditions were as follows: hold for $15 \mathrm{~min}$ at $95^{\circ} \mathrm{C}$, followed by 40 cycles of $60^{\circ} \mathrm{C}$ for $30 \mathrm{sec}$ and $59^{\circ} \mathrm{C}$ for $31 \mathrm{sec}$. The final results were expressed as fold differences in target gene expression related to both the endogenous control gene expression and the calibrator was determined using the $2^{-\Delta \Delta \mathrm{CT}}$ method.

CHOP protein analysis. C6 cells were seeded on a 96-well plate at a density of 3,000 cells/well and exposed to FLT for $24 \mathrm{~h}$. After fixation and permeabilization, $50 \mu \mathrm{l}$ of the mouse monoclonal anti-CHOP antibody (Abcam) solution was added to each sample, which was incubated for $1.5 \mathrm{~h}$ at $37^{\circ} \mathrm{C}$. Next, $50 \mu 1$ of the secondary antibody conjugated with the fluorophore Alexa Fluor ${ }^{\circledR}$ 555/Hoechst solution (Cellomics) was added and incubated at room temperature for $1 \mathrm{~h}$. Then the plate was washed twice with $100 \mu 1$ of blocking buffer. The plate was read on an HCS system reader. The fluorescence intensity represented the level of $\mathrm{CHOP}$ protein in the $\mathrm{C} 6$ cells.

Western blot analysis. C6 cells were cultured on a 6-well plate at a density of $4 \times 10^{5}$ cells/well overnight. Cells were treated with FLT for $24 \mathrm{~h}$. Then we aspirated media, washed the plate with ice-cold 1X PBS for twice and lysed cells with RIPA buffer. Total cell lysates were separated by 10 or $12 \%$ SDS-PAGE and electrotransferred to PVDF membrane (Millipore, Bedford, MA, USA). The membranes were blocked in blocking buffer (5\% nonfat milk in TBS containing $0.1 \%$ Tween-20) for $2 \mathrm{~h}$ at room temperature. After washing in TBST buffer [10 mM Tris-HCl (pH 8.3), 0.05\% Tween-20)] (Tween-20; Sigma-Aldrich, St. Louis, MO, USA), the membranes ware incubated overnight at $4^{\circ} \mathrm{C}$ with the primary antibodies against GADD34 (1:500) and $\beta$-actin $(1: 1,000)$ (both from Santa Cruz Biotechnology) as control. Then the membranes were incubated with horseradish peroxidase-conjugated anti-mouse $\operatorname{IgG}(1: 5,000$; Zhongshan, Beijing,China) at room temperature for $1 \mathrm{~h}$. The immunoblots were visualized using a chemiluminescence detection kit (Pierce Chemical, Rockford, IL, USA).

Small interference RNA experiments. The CHOP-specific siRNAs (5'-UCAAGGAAGAACUAGGAAATT-3' and 5'-UUUCCUAGUUCUUCCUUGATT-3') and scrambled siRNA (5'-UUCUCCGAACGUGUCACGUTT-3' and 5'-ACG UGACACGUUCGGAGAATT-3') were designed and synthesized by Shanghai GenePharma Co., Ltd. (Shanghai, China). C6 cells were plated in a 12-well plate or in a 96-well plate 1 day before transfection. After $60 \%$ confluency was achieved, siRNA was transfected into the culture plates using Lipofectamine 2000 (Invitrogen Life Technologies) following the manufacturer's instructions. Scrambled siRNA was used as a negative control. The knockdown effect was measured by real-time RT-PCR and HCS. Then, the cells were treated with FLT for $24 \mathrm{~h}$. After a 24-h incubation, the cells were washed with medium and used for caspase- 3 activity measurement and CCK-8 assays.

Statistical analysis. Data are presented as means \pm SEM and were analyzed by analysis of variance (ANOVA) followed by Dunnett's test, where $\mathrm{P}<0.05$ indicated a significant difference.

\section{Results}

FLT inhibits the growth of glioma cell lines. FLT is well recognized for its anti-proliferative activity. To determine the effect of FLT on the growth of glioma cells, various glioma cell lines including C6,U87-MG, U373 and U251 glioma cells were used to exam the growth inhibitory ability of FLT by using a CCK-8 assay. All the four glioma cells showed significant inhibition of cell proliferation upon FLT treatment in a dose-dependent manner (Fig. 1A). The $\mathrm{IC}_{50}$ levels of FLT for C6, U87-MG, U373 and U251 cells were 14.7, 21.8, 48.5 and $22.9 \mu \mathrm{M}$, respectively. In particular, C6 cells had a highly sensitive response to the addition of FLT. Thus, C6 cells were selected for further study.

FLT induces apoptotic cell death in C6 glioma cells. To determine whether FLT influences C6 cell apoptosis, we examined the effects of FLT on cell apoptosis. The results showed that FLT treatment for $24 \mathrm{~h}$ decreased the nuclear size of the C6 cells and increased the Hoechst fluorescence intensity in the nuclei in a dose-dependent manner. The nuclei of the C6 cells were significantly condensed at the FLT concentration of $10 \mu \mathrm{M}$ with the concomitant enhanced intensity of Hoechst fluorescence in the nuclei, showing the typical phenotype of apoptosis. This effect was more pronounced with the increase in the drug concentration to above $20 \mu \mathrm{M}$ (Fig. 1B and C). To further determine whether FLT influences cell apoptosis, flow cytometry was performed. As shown in Fig. $2 \mathrm{~A}$ and B, the early apoptosis rate of the C6 cells following FLT $(0,10,20 \mu \mathrm{M})$ treatment by flow cytometry was $6.5,9.21$ and $45.3 \%$, respectively, which demonstrated that FLT significantly increased the percentage of apoptotic cells (Fig. 2A and B). We further assessed the activation of a key apoptosis mediator caspase-3. After FLT treatment, the enzyme activities of caspase-3 in the C6 cells were evidently enhanced (Fig. 2C). These results indicated that FLT can effectively promote the apoptosis of C6 cells.

FLT shows no effect on mitochondrial membrane potential. In order to gain further insight into the mechanism of apoptosis exerted by FLT on C6 cells, we performed a series of experiments in the C6 cells. Since mitochondrial damage represents an event extensively associated with apoptosis, MitoTracker red fluorescence representing MMP was used through the HCS System. Incubation of the C6 cells with FLT did not affect the MitoTracker red fluorescence intensity (Fig. 3). The results showed that FLT may induce cell programmed death through a non-mitochondrial signaling pathway.

FLT induces C6 cell apoptosis through the endoplasmic reticulum apoptotic pathway. Next, we focused on the 
A

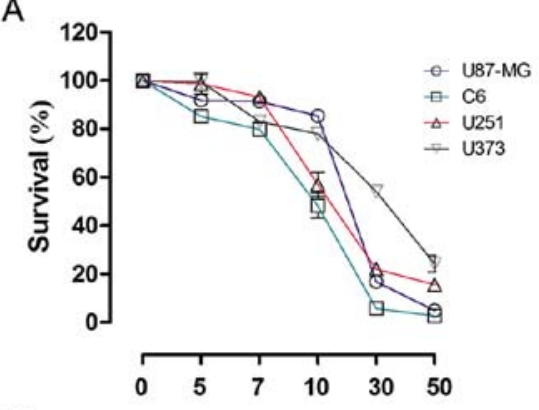

C

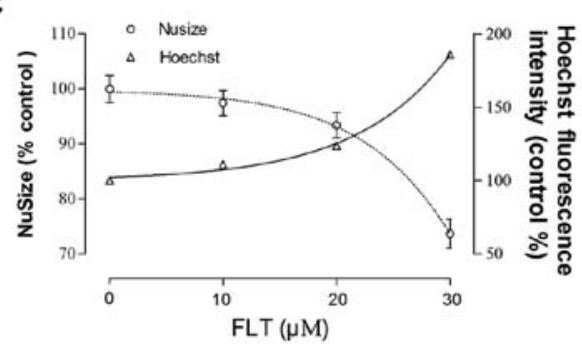

B
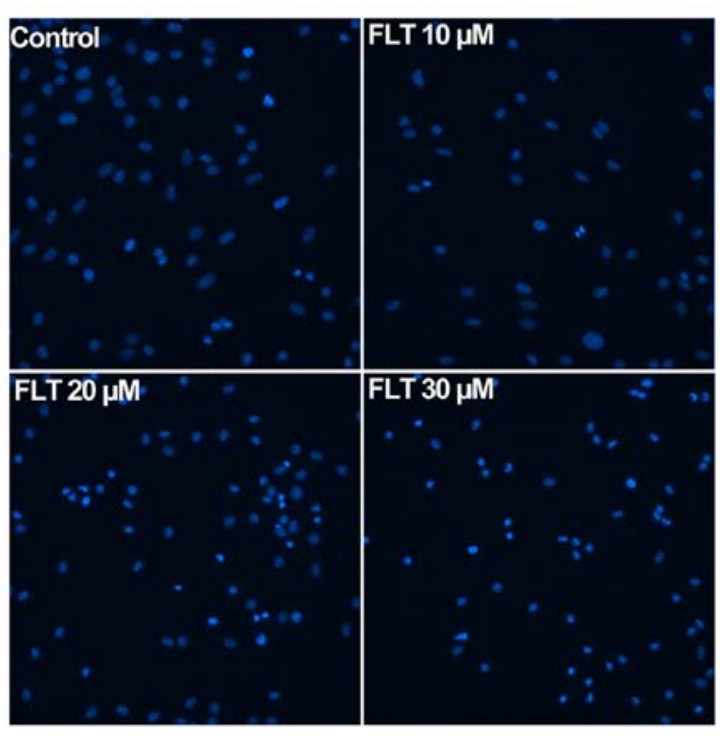

Figure 1. Effect of fluoxetine (FLT) on cell viability and apoptotic death of glioma cell lines. (A) Effect of FLT on cell viability of the glioma cell lines. Cells were incubated with various concentrations of FLT followed by CCK- 8 assays for measuring viability. The data are presented as means \pm SEM from at least three independent experiments. (B) FLT treatment for $24 \mathrm{~h}$ induced the apoptotic death of C6 glioma cells. Detection of apoptosis-specific nuclear morphology with Hoechst dye staining in cell nuclei. (C) The effects of FLT treatment on nuclear size and intensity of Hoechst fluorescence in C6 cells. The data are presented as means \pm SEM from at least three independent experiments.

A
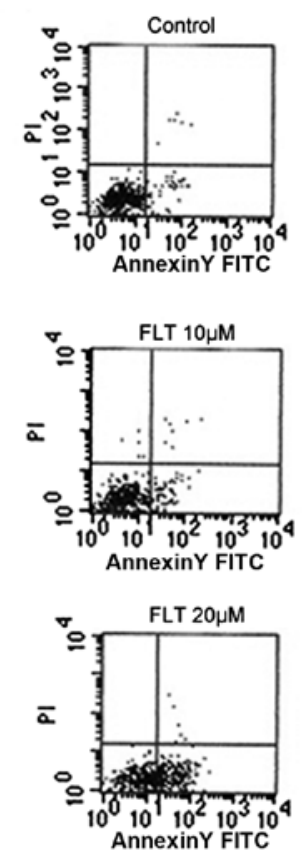
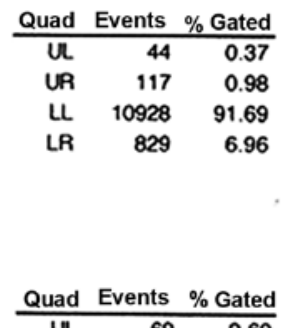

\begin{tabular}{rrr}
\hline UL & 69 & 0.60 \\
UR & 111 & 0.97 \\
UL & 10074 & 88.09 \\
LR & 1182 & 10.34
\end{tabular}

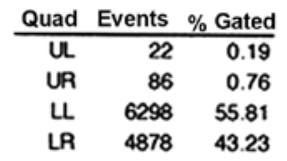

B
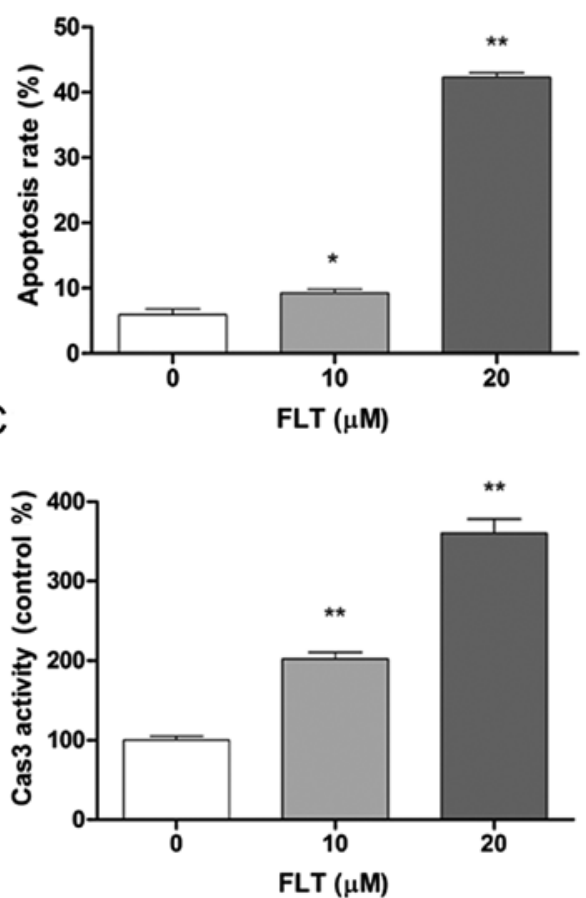

Figure 2. Fluoxetine (FLT) treatment for $24 \mathrm{~h}$ increases the early apoptosis rate and induces caspase-3 activation of C6 glioma cells. Cells were incubated with various concentrations of FLT for $24 \mathrm{~h}$. (A and B) Early apoptosis was measured by the Annexin V assay using flow cytometry. (C) The activity of caspase was determined using a selective luminogenic substrate (Ac-LETD-pNA). The data are presented as means \pm SEM from at least three independent experiments $\left({ }^{*} \mathrm{P}<0.05,{ }^{* *} \mathrm{P}<0.01\right.$ as compared with the control).

endoplasmic reticulum stress (ERS) apoptotic pathway. Firstly we examined the effects of FLT on CHOP, a hallmark of ERS, by real-time RT-PCR, western blotting and HCS, respectively. The results showed that $10 \mu \mathrm{M}$ FLT treatment for $24 \mathrm{~h}$ significantly increased the transcription activation of CHOP mRNA $(\mathrm{P}<0.05)$, and the effect was more pronounced under the higher drug concentration $(20 \mu \mathrm{M})(\mathrm{P}<0.01)$ (Fig. 4A). CHOP protein is present in the cytosol under non-stressed conditions. It can be robustly induced under cell stress conditions and thereafter translocates into nuclei (Fig. 4C). The result of western blotting showed that treatment of FLT $(20 \mu \mathrm{M}, 24 \mathrm{~h})$ significantly increased the expression of CHOP protein (Fig. 4B). 
A

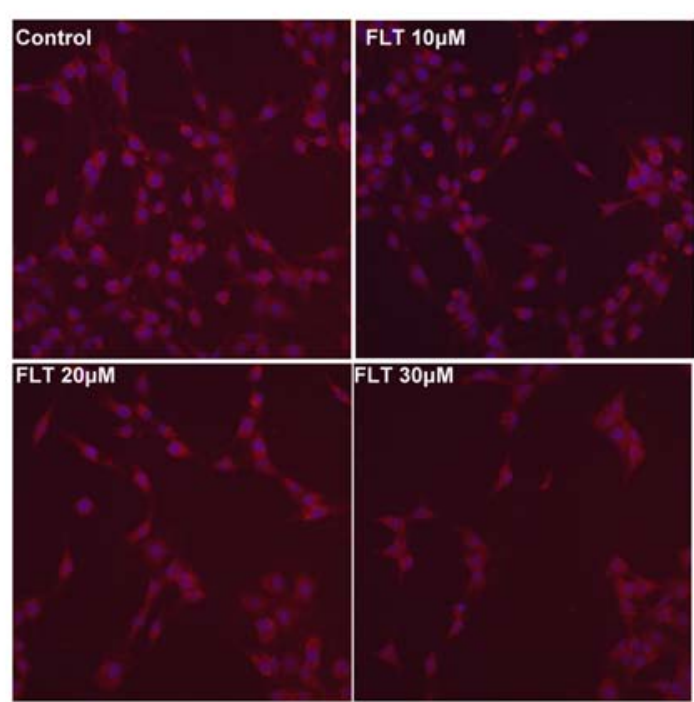

B

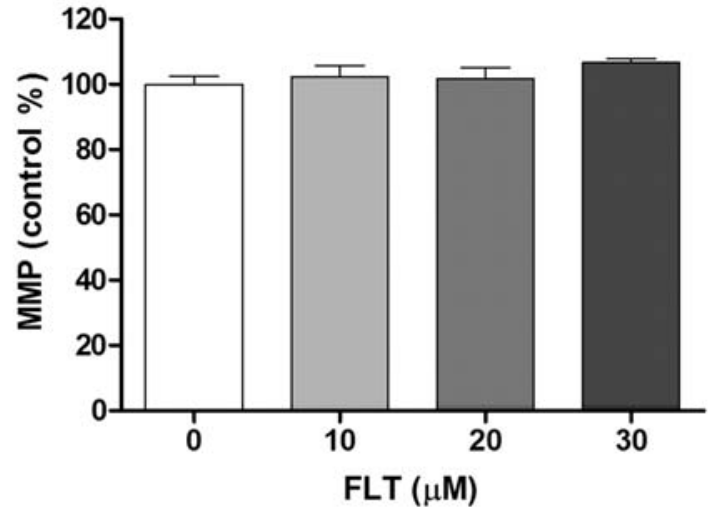

Figure 3. Effect of fluoxetine (FLT) on mitochondrial membrane potential (MMP) in C6 glioma cells. (A) Mitochondrial localization of MitoTracker Red in C6 cells. By the application of MitoTracker Red, red fluorescence occurred in the surrounding mitochondrial membrane. (B) Quantitation of mean fluorescence intensity. The data are presented as means \pm SEM from at least three independent experiments.

A

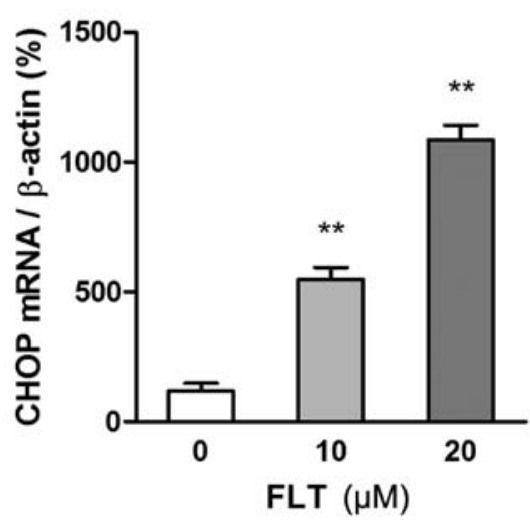

B

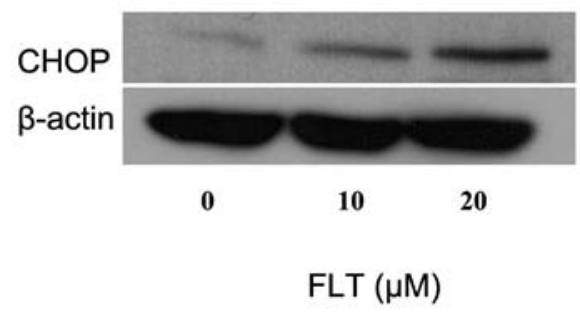

C

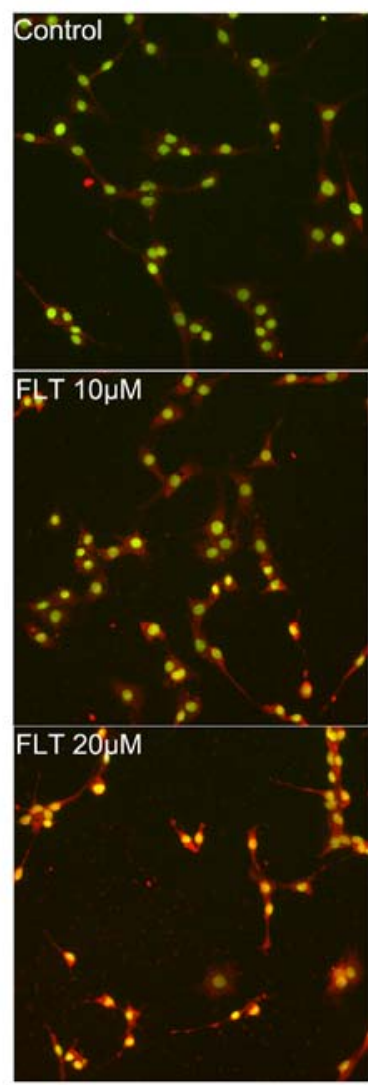

Figure 4. Fluoxetine (FLT) treatment for $24 \mathrm{~h}$ induces CHOP expression. (A) The effect of FLT on the mRNA expression of CHOP in C6 cells. The mRNA expression was determined by quantitative real-time RT-PCR and was standardized to the $\beta$-actin gene. (B) The effect of FLT on the CHOP protein expression in C6 cells. (C) The effects of FLT on the nuclear translocation of CHOP in C6 cells. Color thumbnail images show the color-merged images of nuclear Hoechst (green) and CHOP (red) in the cells, and the yellow areas indicate the co-localization of CHOP protein and nuclei. The data are presented as means \pm SEM from at least three independent experiments $\left({ }^{* *} \mathrm{P}<0.01\right.$ as compared with the control).

Then, we investigated the upstream and downstream signaling pathway of CHOP. The 3 principal unfolded protein response (UPR) receptors involved are PERK, ATF6 and
Ire1, all of which induce expression of CHOP. The protein levels of PERK-eIF2 $\alpha$-ATF4 and ATF6 were examined by western blotting. The results showed that FLT treatment (10 
A

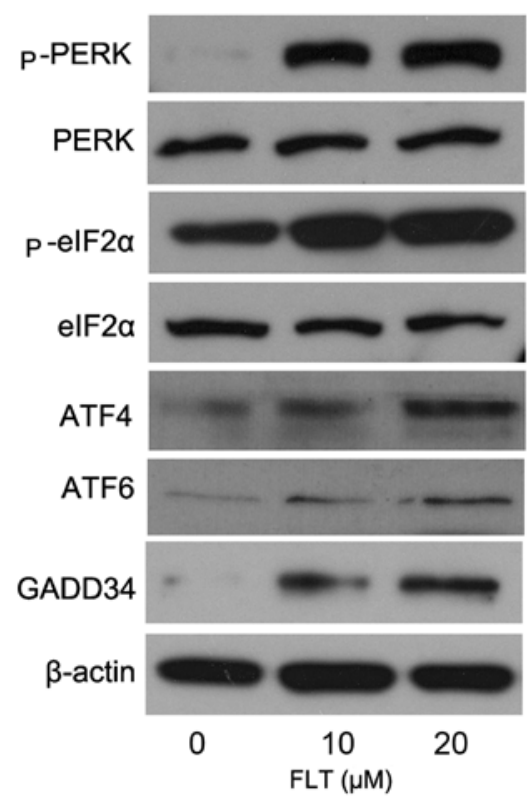

B

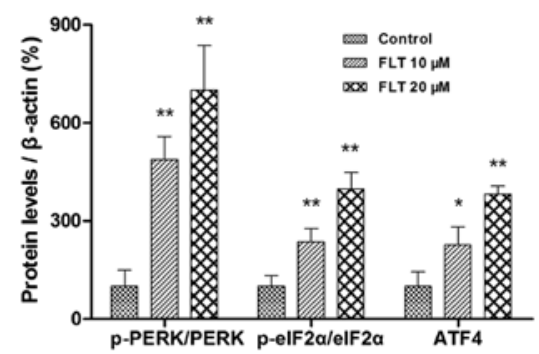

C

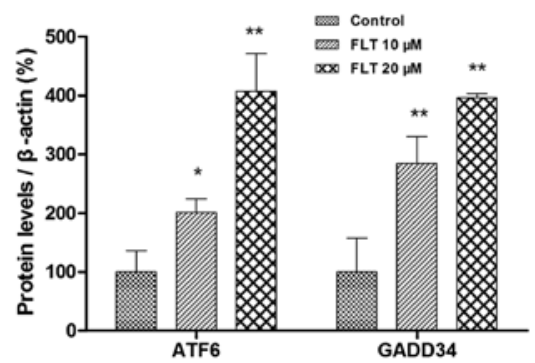

Figure 5. Effect of fluoxetine (FLT) on ER stress sensors in C6 glioma cells. (A) Total proteins were extracted from the C6 cells treated with FLT for 24 h, and were subjected to western blot analyses. (B and C) The densitometric quantitation ratio of ER stress sensors (p-PERK/PERK, p-eIF2 $\alpha /$ eIF2 $\alpha$, ATF4, ATF6 and GADD34) normalized to the $\beta$-actin level. The data are presented as mean $\pm \mathrm{SEM}$ from at least three independent experiments $\left({ }^{*} \mathrm{P}<0.05,{ }^{* *} \mathrm{P}<0.01\right.$ as compared with the control).

and $20 \mu \mathrm{M})$ markedly increased the autophosphorylation of PERK and eIF2 $\alpha$. In addition, ATF4, the downstream target of eIF2 $\alpha$ was increased as well. Meanwhile, another ER stress sensor ATF6 was also markedly upregulated following FLT treatment (Fig. 5). Concomitantly, the protein expression of GADD34, one of the transcriptional downstream effector genes of CHOP activation, was also upregulated (Fig. 5).

FLT sensitizes glioma cells to TMZ by activating CHOP. To explore the possibility of synergistic cytotoxicity, C6 cells were treated with various concentrations of FLT and TMZ, either alone or in combination. Cell viability was determined by CCK- 8 assay and synergistic effects between the two drugs were evaluated using the CompuSyn 2.0 software (ComboSyn, Inc., Paramus, NJ, USA) (23). First, the effect of TMZ alone on the viability of the $\mathrm{C} 6$ cells was examined. As shown in Fig. 6A, TMZ dose-dependently reduced cell viability after a 24-h treatment. In the TMZ treatment group, the 24-h $\mathrm{IC}_{50}$ was $2.23 \mathrm{mM}$. Then, the C6 cells were treated in culture with a combination of FLT and TMZ at various concentrations for $24 \mathrm{~h}$. The combination of FLT and TMZ at certain drug concentrations led to marked effects in decreasing the viability of the C6 cells (Fig. 6B). Synergistic analysis is presented with a Fa-CI plot and CI values (Fig. 6C and D). It was observed that the combination of FLT and TMZ produced a significant synergistic effect and had a CI value below 1.0 (range, 0.736-0.896). The synergism was particularly obvious at lower inhibitory concentrations, whereas little impact was observed at higher proliferation suppression. The synergistic effects of FLT and TMZ were also determined in caspase-3 activity analysis following treatment with TMZ and FLT in the C6 cells (Fig. 6E). Knockdown of CHOP expression by CHOP-specific siRNA was used to further confirm the role of CHOP in the synergistic effect of FLT. CHOPspecific siRNA effectively downregulated CHOP mRNA and protein levels (24). We did not observe enhanced cytotoxicity following treatment with TMZ plus FLT compared to TMZ alone in the C6 cells with CHOP depletion (Fig. 6F). These results suggested that FLT may sensitize glioma cells to TMZ by activating CHOP.

\section{Discussion}

Fluoxetine is one of the most commonly used antidepressants for improving the mood of patients with depressive disorder mainly though inhibiting selective serotonin reuptake. In addition, it is also used to treat chronic pain and other diseases $(25,26)$. Actually, studies have shown that FLT exhibits a series of target-off biological actions such as upregulation of T-cell mediated antitumor immunity, anti-proliferative and pro-apoptotic effects in cancer cells (17-19,27). Since FLT can easily pass through the blood-brain barrier, and has a high concentration in the brain, its direct action on brain tumors is a 'hot' issue. FLT was reported to induce brain tumor apoptosis and even enhance the sensitivity of chemotherapeutic drugs in cancer cells (20). However the underlying mechanisms of apoptosis induced by FLT are not clear.

In the present study, we first found that exposure to FLT significantly and dose-dependently inhibited the proliferation of various glioma cell lines. Among these, C6 cells were most sensitive to FLT treatment. Therefore, whether exposure to FLT would trigger apoptosis was analyzed in C6 cells through subsequent experiments. HCS assay showed that the typical apoptotic morphological indicators such as nuclear shrinkage and DNA condensation appeared in the C6 cells following FLT treatment (Fig. 1). Meanwhile, flow cytometric analysis 
A

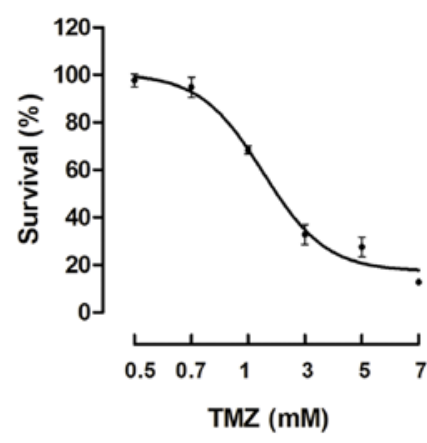

C

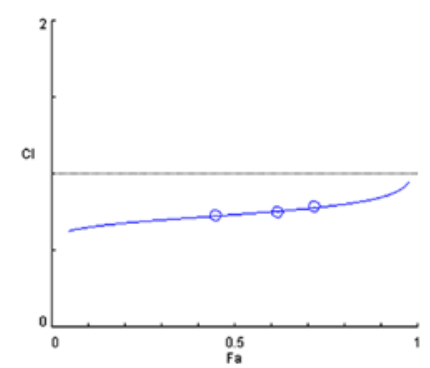

$\mathrm{E}$

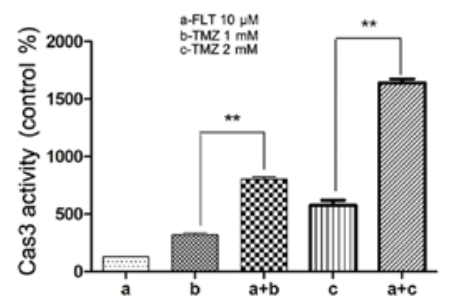

B

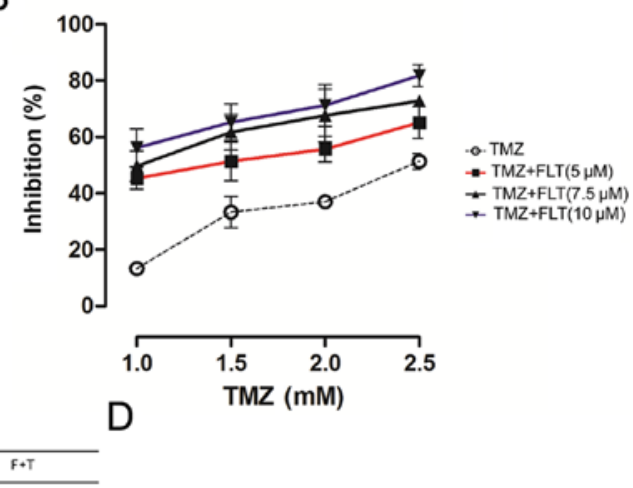

$\mathrm{F}$

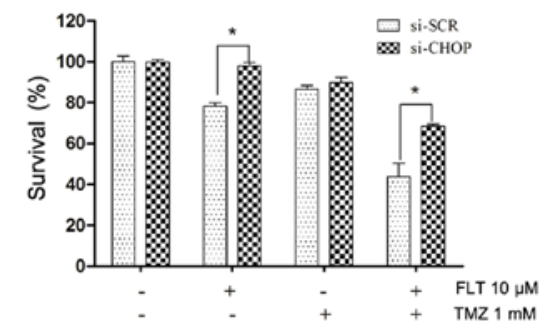

Figure 6. FLT enhances the antitumoral effect of TMZ by activating CHOP. (A) Effect of TMZ on the proliferation of C6 cells. (B) Cytotoxicity analysis of TMZ-induced growth suppression with or without FLT co-treatment in the C6 cells. (C) Synergistic analysis is presented with Fa-CI plot. (D) Combination indices for TMZ and FLT combination therapy, as computed by CompuSyn. (E) Caspase-3 activity analysis following TMZ and FLT in the C6 cells. (F) CHOP knockdown attenuated the synergistic effect of FLT on proliferative inhibition of C6 cells induced by a 24-h treatment of TMZ. The data are presented as means \pm SEM from at least three independent experiments $\left({ }^{*} \mathrm{P}<0.05,{ }^{* *} \mathrm{P}<0.01\right)$. FLT, fluoxetine; TMZ, temozolomide.

also found that FLT induced early apoptotic events in a dosedependent manner, and apoptotic percentage of $20 \mu \mathrm{M}$ FLT was $\sim 45.3 \%$. Simultaneously, caspase-3 activity was greatly activated (Fig. 2), suggesting involvement of the caspasedependent pathway in the pro-apoptotic effect. However, further study found there was no notable change in MMP after FLT treatment, indicating an alternative apoptotic pathway involved in the apoptotic action of FLT. The ERS apoptotic pathway, which differs from the mitochondrial apoptosis pathway, is involved in the apoptotic induction of various anticarcinogens and chemical compounds. Therefore, we next focused on the ERS apoptotic pathway.

The endoplasmic reticulum serves many general functions, including the folding and transport of protein $(28,29)$. Disturbances in calcium regulation, redox regulation, glucose deprivation, and the overexpression of proteins can lead to an endoplasmic reticulum stress response (ER stress) and severe ERS may trigger the apoptosis pathway (30). CHOP is considered to be a crucial regulator of ER stress-related apoptotic signaling (31). Therefore, whether exposure to FLT triggers the ER stress response was analyzed in subsequent experiments. We first determined the effects of FLT on CHOP. We found that a 24-h FLT treatment greatly activated CHOP, which demonstrated that ER stress was elicited by FLT, Then, we further explored the upstream and downstream of the CHOP signaling pathway. The ER stress response triggered under stress conditions is mediated through three main sensors, namely inositol requiring element-1 (IRE-1), protein kinase-like ER kinase (PERK) and activating transcription factor 6 (ATF6) (32). The PERK-eIF2 $\alpha$-ATF4 pathway and ATF6 pathway are the two main branches that activate CHOP. Simultaneously, CHOP mediates cell apoptosis through the induction of genes such as Gadd34 and Erola (32). As expected, western blot results indicated that FLT treatment increased PERK phosphorylation concomitant with the stimulation of eIF $2 \alpha$ phosphorylation, and the levels of ATF4 and ATF6 protein were also increased. Upregulation of Gadd34 expression was also observed in the C6 cells exposed to FLT. All the results indicated that the endoplasmic reticulum (ER) stress-related apoptotic pathway was responsible for FLT-induced apoptosis in the C6 cells. FLT may induce C6 cell apoptosis through the PERKeIF2 $\alpha$-ATF4-Chop and ATF6-Chop signaling pathways.

TMZ is the most effective chemotherapeutic drug in glioma cancer therapy. As a second generation of DNA methylating agents, its cytotoxicity is reported to be mainly mediated through adduction of a methyl group to the $\mathrm{O}^{6}$ position of guanine in genomic DNA. However, more and more experiments support that the antitumor effects induced by TMZ to 
non-DNA targets may also contribute to its action (33-35). Despite the high TMZ potential, progression of disease and recurrence are still observed. It is a key issue to find a strategy by which to enhance the antitumor action of TMZ. A large number of clinical studies have demonstrated that anti-depression treatment in cancer patients not only improves depressive symptoms, but also enhances immunologic function, thereby improving the quality of life of cancer patients. Thus, antidepression treatment has become an important means for adjuvant therapy of cancer. Meanwhile, studies from our and other laboratories have demonstrated that FLT inhibits glioma cell proliferation and induces apoptosis. Therefore, we evaluated whether FLT could improve the sensitivity of C6 glioma cells to the combination chemotherapy. The CI method was used to analyze the type of drug interactions in combination chemotherapy. The combination index (CI) is a parameter that indicates whether the interaction of 2 or more drugs is synergistic $(\mathrm{CI}<1)$, additive $(\mathrm{CI}=1)$, or antagonistic $(\mathrm{CI}>1)$. Our study showed that FLT exerted a synergistic effect on glioma cells when combined with TMZ.

Accumulating data suggest that multi-targeted therapy may produce greater benefits than those observed with single-targeted therapies. The mechanism of FLT may be different from TMZ. Thus, we postulate that FLT as distinguished from TMZ may be partly responsible for their synergistic cytocidal effect in C6 cells. An in vivo study is now being undertaken in our laboratory to evaluate the synergistic effect in inhibiting the growth of tumor xenografts in mice, and we are also trying to identify potential predictive biomarkers with respect to the drug interaction.

To summarize, we showed that FLT is a potential anticancer drug that acts by activating an ERS-related apoptotic pathway in the treatment of glioma. In addition, our findings suggest that FLT exerts a synergistic effect on glioma cells when combined with TMZ. Further studies are needed to elucidate the role of the ERS-related apoptotic pathway in the synergistic effect of FLT and TMZ.

\section{Acknowledgements}

We gratefully thank the National Natural Science Foundation of China (nos. 81302202, 81272683, 81441111) and the Natural Science Foundation of Shandong Province (no.ZR2011HQ055) for the financial support.

\section{References}

1. Goodenberger ML and Jenkins RB: Genetics of adult glioma Cancer Genet 205: 613-621, 2012.

2. Ohgaki H and Kleihues P: Epidemiology and etiology of gliomas. Acta Neuropathol 109: 93-108, 2005.

3. Wang F, Huang Q and Zhou LY: Analysis of the treatment of gliomas with SEC therapy combined with radiochemotherapy. Eur Rev Med Pharmacol Sci 19: 2400-2405, 2015.

4. Quick A, Patel D, Hadziahmetovic M, Chakravarti A and Mehta M: Current therapeutic paradigms in glioblastoma. Rev Recent Clin Trials 5: 14-27, 2010.

5. Stupp R, Mason WP, van den Bent MJ, Weller M, Fisher B, Taphoorn MJ, Belanger K, Brandes AA, Marosi C, Bogdahn U, et al; European Organisation for Research and Treatment of Cancer Brain Tumor and Radiotherapy Groups; National Cancer Institute of Canada Clinical Trials Group: Radiotherapy plus concomitant and adjuvant temozolomide for glioblastoma. $\mathrm{N}$ Engl J Med 352: 987-996, 2005.
6. Stupp R, Hegi ME, Mason WP, van den Bent MJ, Taphoorn MJ, Janzer RC, Ludwin SK, Allgeier A, Fisher B, Belanger K, et al; European Organisation for Research and Treatment of Cancer Brain Tumour and Radiation Oncology Groups; National Cancer Institute of Canada Clinical Trials Group: Effects of radiotherapy with concomitant and adjuvant temozolomide versus radiotherapy alone on survival in glioblastoma in a randomised phase III study: 5-year analysis of the EORTC-NCIC trial. Lancet Oncol 10: 459-466, 2009.

7. Neyns B, Tosoni A, Hwu WJ and Reardon DA: Dose-dense temozolomide regimens: Antitumor activity, toxicity, and immunomodulatory effects. Cancer 116: 2868-2877, 2010.

8. Hori YS, Hosoda R, Akiyama Y, Sebori R, Wanibuchi M, Mikami T, Sugino T, Suzuki K, Maruyama M, Tsukamoto M, et al: Chloroquine potentiates temozolomide cytotoxicity by inhibiting mitochondrial autophagy in glioma cells. J Neurooncol 122: 11-20, 2015.

9. Zhang Y, Wang SX, Ma JW, Li HY, Ye JC, Xie SM, Du B and Zhong XY: EGCG inhibits properties of glioma stem-like cells and synergizes with temozolomide through downregulation of P-glycoprotein inhibition. J Neurooncol 121: 41-52, 2015.

10. Sheehan J, Cifarelli CP, Dassoulas K, Olson C, Rainey J and Han S: Trans-sodium crocetinate enhancing survival and glioma response on magnetic resonance imaging to radiation and temozolomide. J Neurosurg 113: 234-239, 2010.

11. Park EM and Rosenstein DL: Depression in adolescents and young adults with cancer. Dialogues Clin Neurosci 17: 171-180, 2015 .

12. Lauer AL: Treatment of anxiety and depression in adolescents and young adults with cancer. J Pediatr Oncol Nurs 32: 278-283, 2015.

13. Perez-Caballero L, Torres-Sanchez S, Bravo L, Mico JA and Berrocoso E: Fluoxetine: A case history of its discovery and preclinical development. Expert Opin Drug Discov 9: 567-578, 2014.

14. Kannen V, Marini T, Turatti A, Carvalho MC, Brandão ML, Jabor VA, Bonato PS, Ferreira FR, Zanette DL, Silva WA Jr, et al: Fluoxetine induces preventive and complex effects against colon cancer development in epithelial and stromal areas in rats. Toxicol Lett 204: 134-140, 2011.

15. Koh SJ, Kim JM, Kim IK, Kim N, Jung HC, Song IS and Kim JS: Fluoxetine inhibits NF- $\kappa$ B signaling in intestinal epithelial cells and ameliorates experimental colitis and colitis-associated colon cancer in mice. Am J Physiol Gastrointest Liver Physiol 301: G9-G19, 2011.

16. Kannen V, Hintzsche H, Zanette DL, Silva WA Jr, Garcia SB, Waaga-Gasser AM and Stopper H: Antiproliferative effects of fluoxetine on colon cancer cells and in a colonic carcinogen mouse model. PLoS One 7: e50043, 2012.

17. Mun AR, Lee SJ, Kim GB, Kang HS, Kim JS and Kim SJ: Fluoxetine-induced apoptosis in hepatocellular carcinoma cells. Anticancer Res 33: 3691-3697, 2013.

18. Lin KL, Chou CT, Cheng JS, Chang HT, Liang WZ, Kuo CC, Chen IL, Tseng LL, Shieh P, Wu RF, et al: Effect of fluoxetine on $\left[\mathrm{Ca}^{2+}\right] \mathrm{i}$ and cell viability in $\mathrm{OC} 2$ human oral cancer cells. Chin J Physiol 57: 256-264, 2014.

19. Lee CS, Kim YJ, Jang ER, Kim W and Myung SC: Fluoxetine induces apoptosis in ovarian carcinoma cell line OVCAR-3 through reactive oxygen species-dependent activation of nuclear factor-kappaB. Basic Clin Pharmacol Toxicol 106: 446-453, 2010.

20. Song T, Li H, Tian Z, Xu C, Liu J and Guo Y: Disruption of NF- $\kappa \mathrm{B}$ signaling by fluoxetine attenuates MGMT expression in glioma cells. Onco Targets Ther 8: 2199-2208, 2015.

21. Cloonan SM and Williams DC: The antidepressants maprotiline and fluoxetine induce Type II autophagic cell death in drugresistant Burkitt's lymphoma. Int J Cancer 128: 1712-1723, 2011.

22. Zhou T, Duan J, Wang Y, Chen X, Zhou G, Wang R, Fu L and $\mathrm{Xu}$ F: Fluoxetine synergys with anticancer drugs to overcome multidrug resistance in breast cancer cells. Tumour Biol 33: 1299-1306, 2012.

23. Bijnsdorp IV, Giovannetti E and Peters GJ: Analysis of drug interactions. Methods Mol Biol 731: 421-434, 2011.

24. Ma J, Qiu Y, Yang L, Peng L, Xia Z, Hou LN, Fang C, Qi H and Chen HZ: Desipramine induces apoptosis in rat glioma cells via endoplasmic reticulum stress-dependent CHOP pathway. J Neurooncol 101: 41-48, 2011.

25. Bundeff AW and Woodis CB: Selective serotonin reuptake inhibitors for the treatment of irritable bowel syndrome. Ann Pharmacother 48: 777-784, 2014. 
26. Dharmshaktu P, Tayal V and Kalra BS: Efficacy of antidepressants as analgesics: A review. J Clin Pharmacol 52: 6-17, 2012.

27. Frick LR, Rapanelli M, Arcos ML, Cremaschi GA and Genaro AM: Oral administration of fluoxetine alters the proliferation/apoptosis balance of lymphoma cells and up-regulates $\mathrm{T}$ cell immunity in tumor-bearing mice. Eur J Pharmacol 659: 265-272, 2011.

28. Koch GL: The endoplasmic reticulum and calcium storage. BioEssays 12: 527-531, 1990.

29. Klausner RD and Sitia R: Protein degradation in the endoplasmic reticulum. Cell 62: 611-614, 1990.

30. $\mathrm{Wu} \mathrm{H}, \mathrm{Ng}$ BS and Thibault G: Endoplasmic reticulum stress response in yeast and humans. Biosci Rep 34: e00118, 2014.

31. Oyadomari S and Mori M: Roles of CHOP/GADD153 in endoplasmic reticulum stress. Cell Death Differ 11: 381-389, 2004.
32. Sano R and Reed JC: ER stress-induced cell death mechanisms. Biochim Biophys Acta 1833: 3460-3470, 2013.

33. Roos WP, Batista LF, Naumann SC, Wick W, Weller M, Menck CF and Kaina B: Apoptosis in malignant glioma cells triggered by the temozolomide-induced DNA lesion $\mathrm{O}^{6}$-methylguanine. Oncogene 26: 186-197, 2007.

34. Zhang WB, Wang Z, Shu F, Jin YH, Liu HY, Wang QJ and Yang Y: Activation of AMP-activated protein kinase by temozolomide contributes to apoptosis in glioblastoma cells via p53 activation and mTORC1 inhibition. J Biol Chem 285: 40461-40471, 2010.

35. Zou Y, Wang Q and Wang W: MutL homolog 1 contributes to temozolomide-induced autophagy via ataxia-telangiectasia mutated in glioma. Mol Med Rep 11: 4591-4596, 2015 